\title{
SwissDRG: Benachteiligung in der Abgeltung multimorbider Patienten
}

Thomas Beck $k^{a, h}$, Christine Gersching ${ }^{b, h}$, Karin Fattinger ${ }^{c, h}$, Kerstin Schlimbach Neuhauser ${ }^{d, h}$, Georg Mange,h, Jörg Leuppi ${ }^{f, h}$, Bernadette Häfliger Berger ${ }^{g}$, Drahomir Aujeskya , Jacques Donzéa,

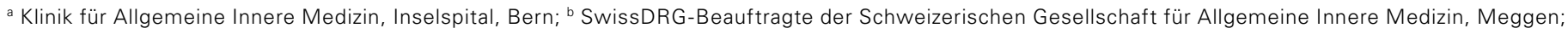

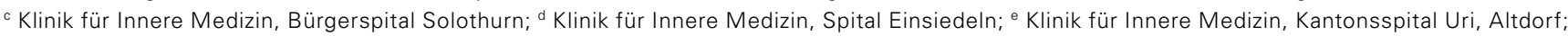

${ }^{\dagger}$ Medizinische Universitätsklinik, Kantonsspital Baselland, Liestal; ${ }^{9}$ Generalsekretärin der Schweizerischen Gesellschaft für Allgemeine Innere Medizin, Bern;

${ }^{\text {h }}$ Mitglieder des SwissDRG-Panels der SGAIM

\section{Einführung}

Multimorbidität wird definiert als gleichzeitiges Vorliegen von zwei oder mehr chronischen oder akuten Erkrankungen und gesundheitlichen Störungen bei einer einzelnen Person [1]. Ein Patient mit mehr als 4 Krankheiten hat 3,2-mal mehr ambulante Konsultationen, wird 4,5-mal häufiger hospitalisiert und verursacht durchschnittlich 5-mal höhere Kosten pro Jahr als ein Patient ohne chronische Krankheit [2]. Multimorbide Patienten haben eine schlech-

\section{Résumé}

Le nombre de patients multimorbides augmente dans le monde entier, notamment avec l'allongement de l'espérance de vie.

La Société Suisse de Médecine Interne Générale nous a chargés de vérifier si le traitement des patients multimorbides était rémunéré de façon appropriée dans le système SwissDRG. Pour cela, nous nous sommes appuyés sur une analyse sur quatre ans de près $d^{\prime} 1,5$ million de cas, dans toutes les catégories d'hôpitaux et toutes les régions du pays.

Dans le système DRG, les patients présentant des comorbidités ou des complications sévères ou extrêmement sévères constituent à peine un cinquième de nos données. Notre analyse montre que, dans notre collectivité, d'une part les cas de multimorbidité, d'autre part les forfaits par cas avec une forte proportion de cas de multimorbidité sont nettement désavantagés du point de vue économique.

Cela peut entraîner des incitations négatives et compromettre les soins des patients multimorbides.

Ne considérer que le taux de couverture des coûts, semblable à celui des cas moins complexes et présentant une moins grande multimorbidité, peut aboutir à des conclusions erronées. Ce qui est déterminant pour le succès d'un hôpital, ce n'est pas le taux de couverture relatif des coûts, mais le résultat absolu et les coûts nettement plus élevés qu'il entraîne pour les patients multimorbides.

Là aussi, à la demande de la Société Suisse de Médecine Interne Générale, SwissDRG SA consacrera un point de développement prioritaire à cette thématique dans la prochaine version du système (7.0). tere Lebensqualität und ein höheres Sterberisiko (Hazard Ratio 1,73 bei zwei bzw. 2,72 bei drei Erkrankungen) $[3,4]$.

Ein Patient mit mehr als 4 Krankheiten verursacht durchschnittlich 5-mal höhere Kosten pro Jahr als ein Patient ohne chronische Krankheit.

Insgesamt sind 23\% aller Personen multimorbid, über 65 Jahre sind es sogar knapp 65\% [5]. Durch die steigende Lebenserwartung und die Fortschritte in medizinischer Behandlung und Prävention werden multimorbide Patienten weltweit immer häufiger [6]. In der Schweiz wird von 2015 bis 2045 mit einem Bevölkerungszuwachs um 1,9 Mio. auf knapp 10,2 Mio. Personen gerechnet. Das prognostizierte Bevölkerungswachstum betrifft überproportional die Altersgruppe der über 65-Jährigen, deren Zahl allein um 1,2 Mio. Personen zunehmen wird [7]. Daraus lässt sich schätzen, dass bis in knapp 30 Jahren in der Schweiz die Zahl multimorbider Patienten um ca. 800000 Personen auf gut 2,3 Mio. ansteigen wird.

Multimorbide Patienten sind somit aufwendig in der Behandlung und werden in naher Zukunft deutlich häufiger behandelt werden müssen. Wir stellten uns hier die Frage, ob multimorbide Fälle unter SwissDRG adäquat abgebildet und vergütet werden.

\section{Abbildung der Multimorbidität in den DRGs}

Die DRGs werden primär durch die Hauptdiagnose und allfällige signifikante Prozeduren definiert. Es liegt auf der Hand, dass multimorbide Patienten mit der gleichen Hauptdiagnose einen höheren Behandlungsaufwand aufweisen als solche ohne Komorbiditäten. Bei vielen DRGs werden deshalb Komorbiditäten und Komplikationen berücksichtigt, indem multimorbide Fälle innerhalb einer Basis-DRG (z.B. E77 Andere Infek- 
tionen und Entzündungen der Atemwege) in eine Unter-DRG eingeordnet werden, die höher vergütet wird und eine höhere mittlere Aufenthaltsdauer aufweist. Der Schweregrad der Komorbiditäten und/oder Komplikationen $(\mathrm{CC})$ jedes Falls wird mit einem "patient

Der Schweregrad der Komorbiditäten und/oder Komplikationen (CC) jedes Falls wird mit einem "patient complexity and complications level» (PCCL) angegeben.

complexity and complications level» (PCCL) angegeben. Der PCCL wird in einem komplizierten Verfahren anhand der Nebendiagnosen eruiert. Die Nebendiagnosen werden dabei nicht einfach addiert, sondern abhängig von der Hauptdiagnose und allfälligen anderen Nebendiagnosen gewichtet. Anhand einer komplexen Formel wird der PCCL berechnet und in fünf Schweregrade unterteilt: 0 bedeutet keine signifikanten Komorbiditäten oder Komplikationen, 4 bedeutet äusserst schwere Komorbiditäten oder Komplikationen.

\section{Hypothesen}

Unserer Arbeit liegt die Annahme zugrunde, dass der erhöhte Behandlungsaufwand von multimorbiden Patienten mit hohem PCCL unter SwissDRG nicht adäquat vergütet wird. Unsere Hypothesen waren, dass: 1. multimorbide Fälle mit einem hohen PCCL im Durchschnitt ökonomisch benachteiligt werden (nachfolgend Fallsicht) und/oder

2. DRGs mit einem hohen durchschnittlichen PCCLWert, der einem hohen Anteil an multimorbiden Fällen in dieser DRG gleichzusetzen ist, ökonomisch benachteiligt werden (nachfolgend DRG-Sicht).

\section{Methode}

Um diese Hypothesen datengestützt zu prüfen, hat die Schweizerische Gesellschaft für Allgemeine Innere Medizin (SGAIM) ab dem Jahr 2013 anonymisierte medizinische und Kostenträger-Datensätze gesammelt

\section{Um die Hypothesen zu prüfen, hat die SGAIM anonymisierte Datensätze gesammelt.}

und ausgewertet. Die zusammengeführten Resultate wurden mit Unterstützung eines Expertengremiums (SwissDRG-Panel) aus den Reihen der SGAIM analysiert. Wir haben die Fälle deshalb wie folgt kategorisiert:
1. Fallsicht: Einem Fall wird bei jedem Spitalaufenthalt entsprechend seinen Komorbiditäten ein PCCL-Wert zwischen 0 und 4 zugeordnet.

2. DRG-Sicht: Die DRGs enthalten viele Fälle, die jeweils einen individuellen PCCL-Wert haben. Der durchschnittliche PCCL-Wert einer DRG entspricht dem durchschnittlichen PCCL aller in ihr enthaltenen Fälle. Die DRGs wurden in 4 Kategorien mit steigendem Anteil an multimorbiden Fällen eingeteilt (PCCL 0 bis 0,99, 1 bis 1,99, 2 bis 2,99 und 3,0 bis 4,0). Im Gegensatz zu der Fallsicht kann die Unterteilung nicht ganzzahlig erfolgen, weil ca. zwei Drittel der DRGs Fälle mit unterschiedlichem PCCL in sich vereinen und/oder nicht primär durch die Multimorbidität, sondern beispielsweise durch einen operativen Eingriff oder eine lange Beatmungszeit definiert sind.

\section{Der durchschnittliche PCCL-Wert einer DRG entspricht dem durchschnittlichen PCCL aller in ihr enthaltenen Fälle.}

Für beide Sichtweisen wurde für jede PCCL-Kategorie berechnet:

- Das durchschnittliche finanzielle Ergebnis pro Fall. Dies entspricht der Summe der Erlöse minus der Summe der Fallkosten geteilt durch die Fallzahl und wird in Franken (Fr.) angegeben. Hier handelt es sich um einen absoluten Wert.

- Der durchschnittliche Kostendeckungsgrad. Dies entspricht der Summe der Erlöse geteilt durch die Summe der Fallkosten in Prozent. Hier handelt es sich um einen relativen Wert. Liegt er über 100\%, resultiert ein Gewinn, liegt er unter 100\%, resultiert ein Verlust. Selbst wenn der Wert nahe bei 100\% liegt, können sich je nach Höhe der Fallkosten sehr unterschiedliche absolute Gewinne oder Verluste ergeben.

- Der prozentuale Anteil der Fälle in den verschiedenen Kategorien.

\section{Resultate}

Es standen 1482630 bereits plausibilisierte Fälle von Januar 2013 bis Dezember 2016 zur Verfügung, was gut einem Viertel bis knapp einem Drittel der an die SwissDRG AG gelieferten Datensätze entspricht. Die Daten stammten je nach Jahr von 22 bis 31 Spitälern aller Versorgungsstufen und aus allen Landesteilen, darunter zwei Privatspitäler. Unter den 31 beteiligten Spitälern befinden sich drei Universitätsspitäler, 15 nichtuniversitäre Zentrumsspitäler und 13 Kliniken der Grundversorgung (gemäss Krankenhaustypologie des Bundesamts für Statistik BFS). 


\section{Fallsicht}

Das durchschnittliche Ergebnis pro Fall sank in allen vier Jahren mit steigendem PCCL, wobei der durchschnittliche Verlust von knapp 2600 auf knapp 4500 Franken bei PCCL 4 ansteigt (Abb. 1a).

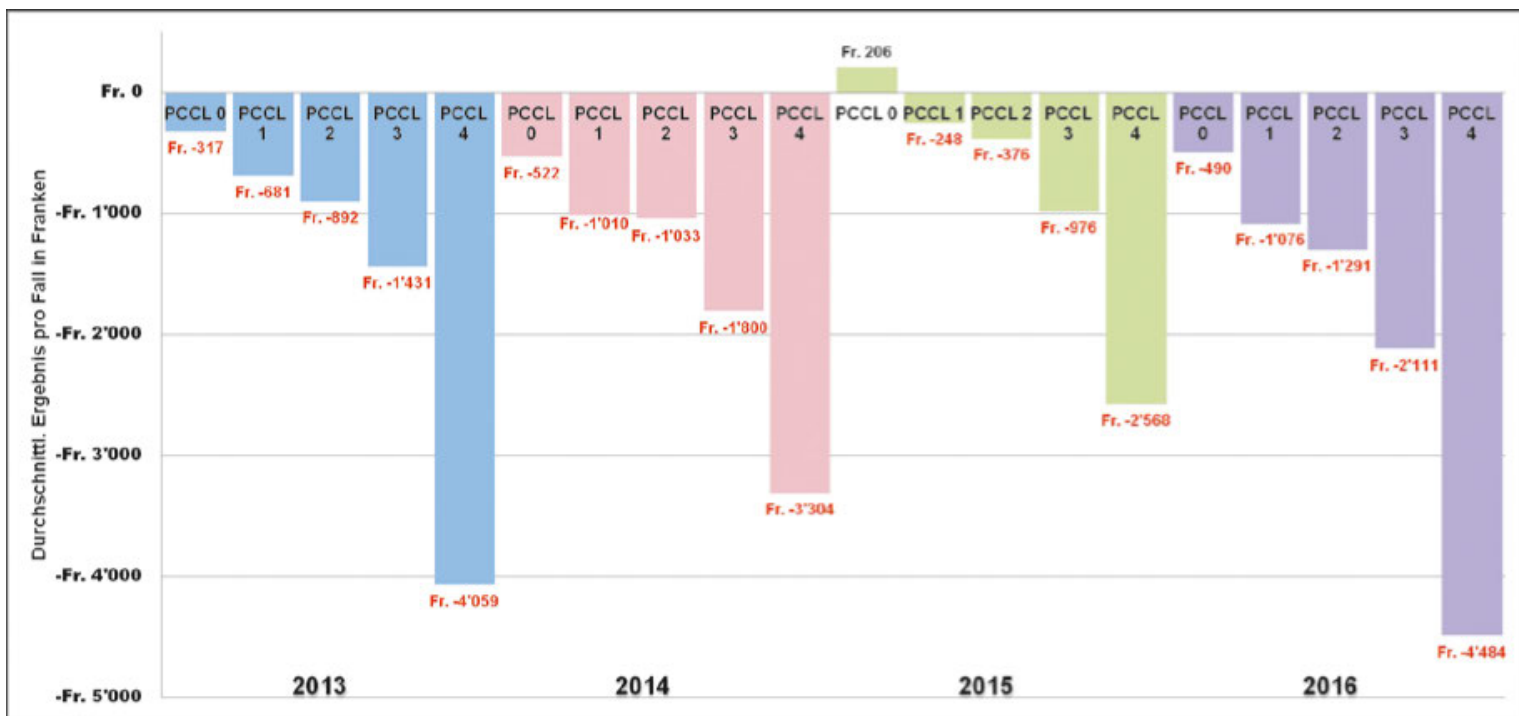

Abbildung 1a: Durchschnittliches Ergebnis mit ansteigendem PCCL (Fallsicht).

$\mathrm{PCCL}=$ patient complexity and complications level

Der Kostendeckungsgrad war in den PCCL-Kategorien 3 und 4 immer tiefer als in den Kategorien 0 bis 2 (Abb. 1b).

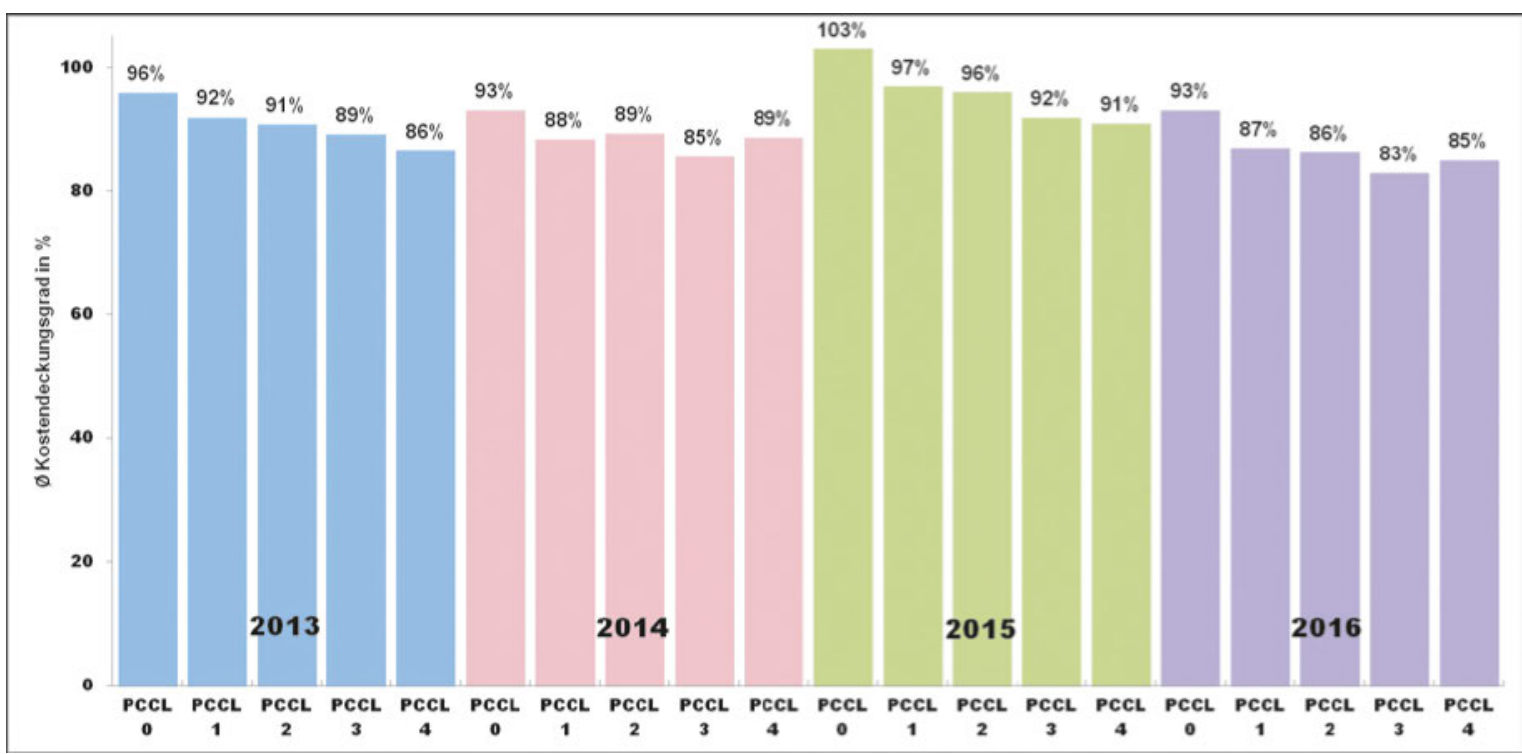

Abbildung 1b: Durchschnittlicher Kostendeckungsgrad mit ansteigendem PCCL (Fallsicht).

$\mathrm{PCCL}=$ patient complexity and complications level

Die prozentuale Verteilung der Fälle auf die verschiedenen PCCL-Kategorien war über die Jahre weitgehend konstant mit einem kumulierten Anteil der Fälle mit schweren und äusserst schweren Komorbiditäten und Komplikationen (PCCL 3 und 4) von ca. 25\% (Abb. 1c auf der folgenden Seite). 


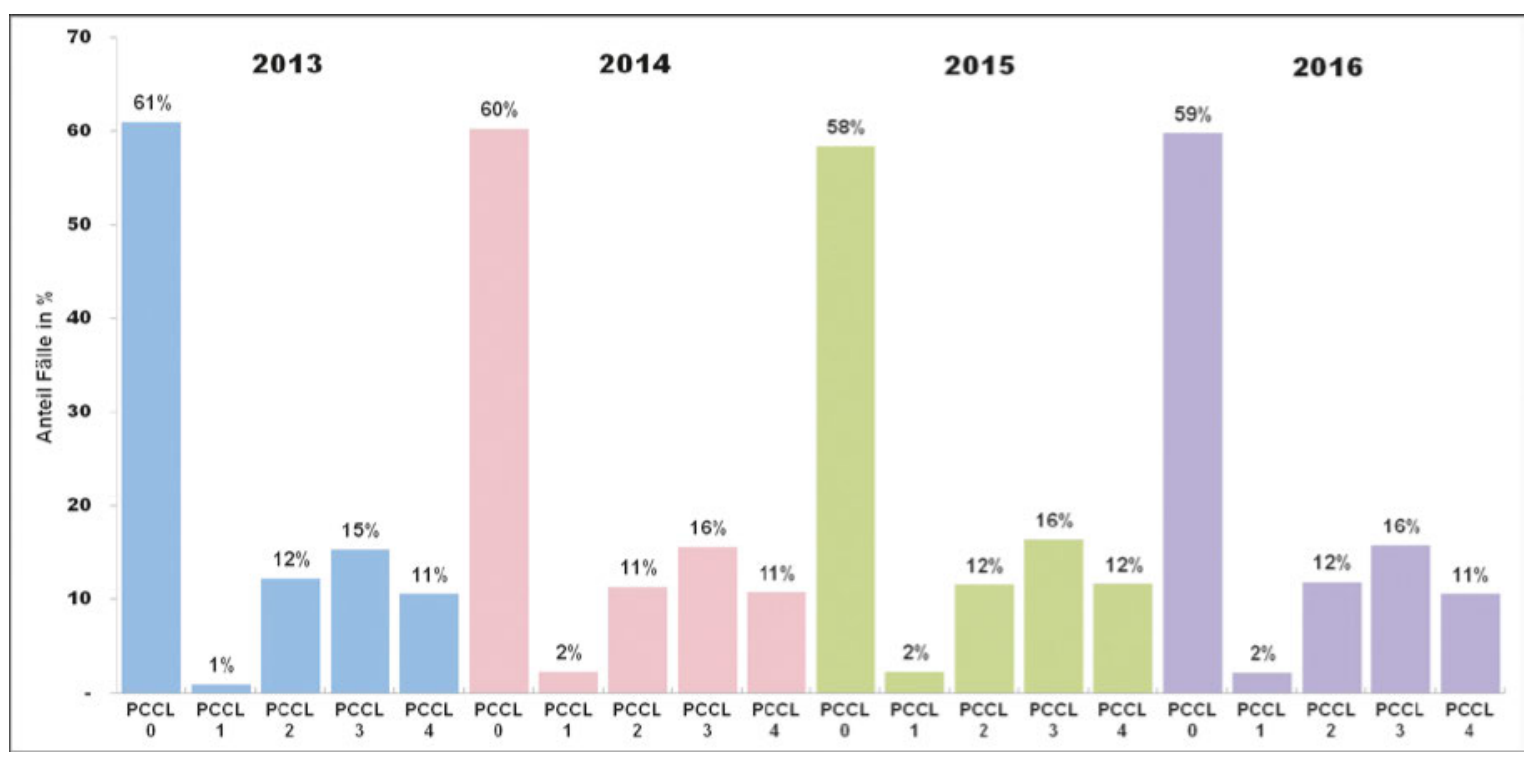

Abbildung 1c: Prozentualer Anteil der Fälle (Fallsicht).

$\mathrm{PCCL}=$ patient complexity and complications level

\section{DRG-Sicht}

Das durchschnittliche Defizit pro Fall innerhalb der DRGs nahm in allen vier Jahren mit steigender PCCL-Kategorie zu und war in der Kategorie PCCL 3,0 bis 4,0 im Vergleich zur Kategorie 0 bis 0,99 knapp zwei- bis über dreimal höher mit einem Defizit von bis zu 3000 Franken pro Fall (Abb. 2a).

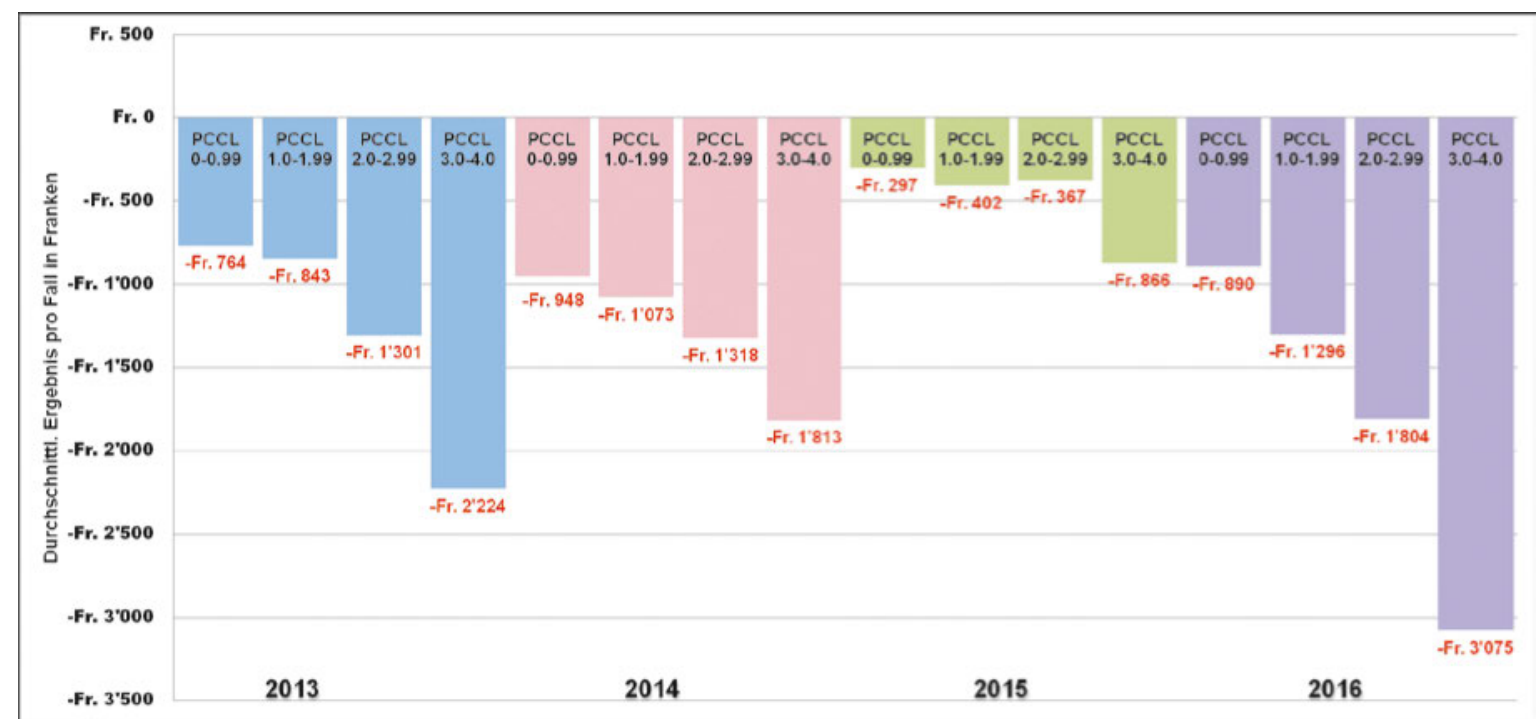

Abbildung 2a: Durchschnittliches Ergebnis pro Fall (DRG-Sicht).

$\mathrm{PCCL}=$ patient complexity and complications level

Der Kostendeckungsgrad war bei allen PCCL-Kategorien ähnlich (89\% bis 98\%), in den Jahren 2014 und 2016 lag er in der PCCL-Kategorie 3,0 bis 4,0 etwas über den anderen Kategorien (Abb. 2b auf der folgenden Seite). 


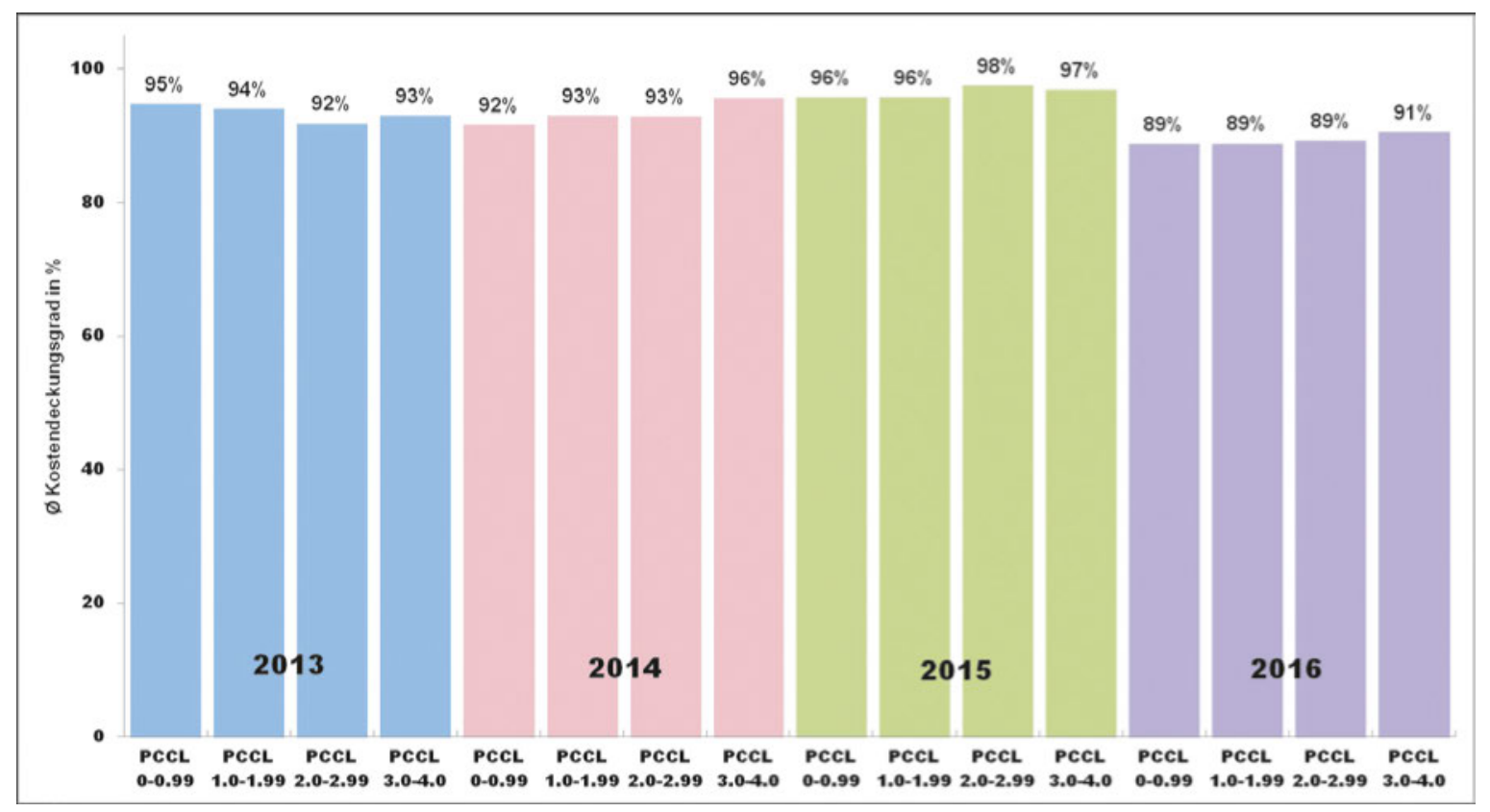

Abbildung 2b: Durchschnittlicher Kostendeckungsgrad (DRG-Sicht). PCCL = patient complexity and complications level

Die prozentuale Verteilung der Fälle innerhalb der DRG-Kategorien war über die Jahre weitgehend konstant mit einem kumulierten Anteil der Fälle mit schweren und äusserst schweren Komorbiditäten und Komplikationen (PCCL 3 und 4) zwischen knapp 17\% und knapp 20\% (Abb. 2c).

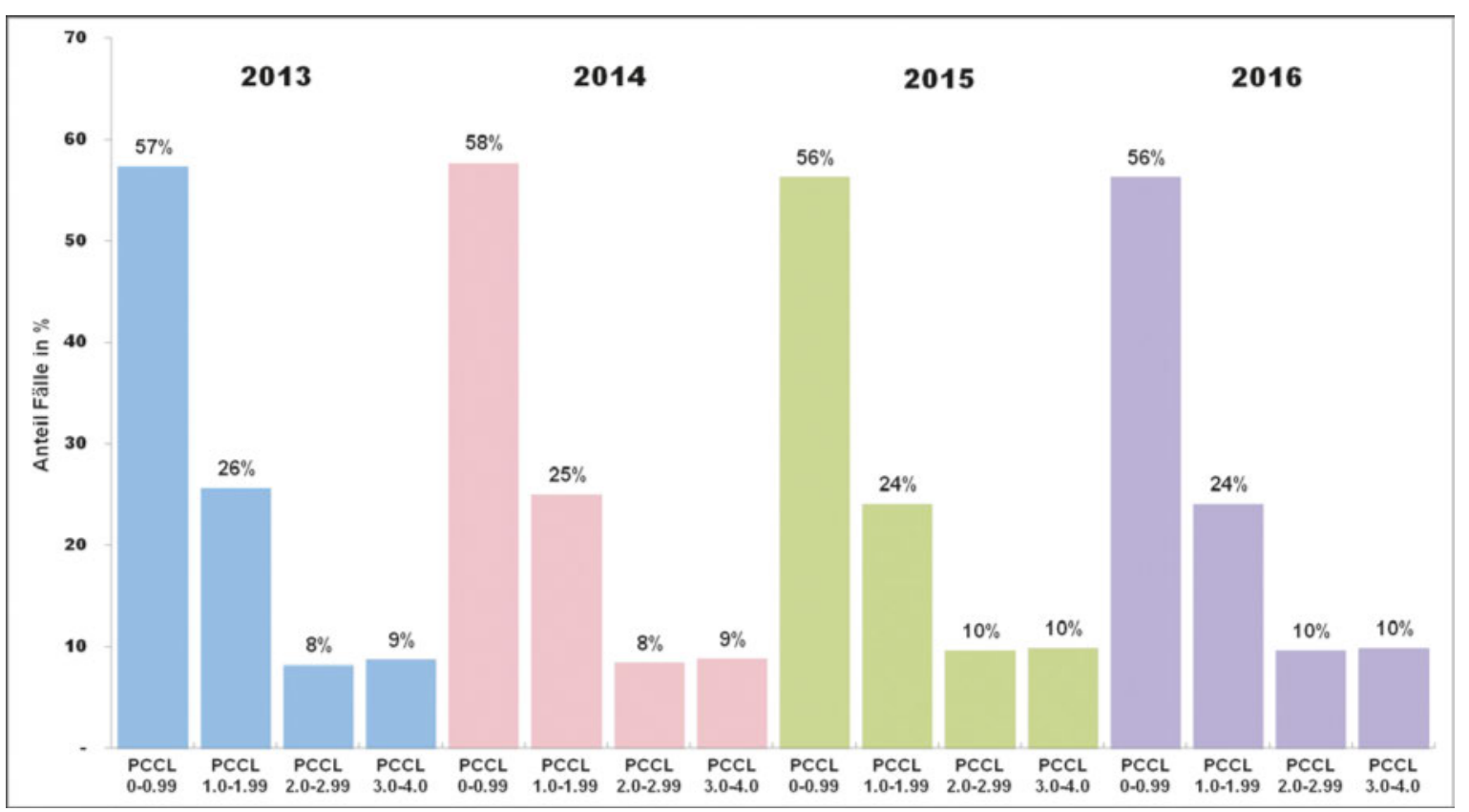

Abbildung 2c: Prozentualer Anteil der Fälle (DRG-Sicht).

$\mathrm{PCCL}=$ patient complexity and complications level

Subgruppenanalysen mit der gleichen Methodik der Hauptkostenstelle Innere Medizin (M100) und der drei Partitionen ( $\mathrm{M}=$ medizinisch, $\mathrm{O}=$ operativ und $\mathrm{A}=$ andere) zeigten sowohl bezüglich der Fallsicht wie auch der DRG-Sicht ähnliche Resultate.

\section{Diskussion}

Aus unserer umfangreichen Datenbasis mit kumulativ fast 1,5 Mio. Fällen über vier Jahre lässt sich erkennen, dass die multimorbiden Fälle derzeit ökonomisch benachteiligt werden, indem das finanzielle Ergebnis 
reziprok mit dem PCCL-Wert und damit dem Grad der Multimorbidität korreliert. Dieser Effekt lässt sich von 2013 bis 2016 konstant nachweisen.

Dadurch können Fehlanreize entstehen, da es für Spitäler oder Spitalabteilungen aus ökonomischer Sicht attraktiver sein kann, bevorzugt weniger komplexe Patienten zu behandeln. Umgekehrt sind Spitäler mit vielen multimorbiden Fällen auf höhere Baserates angewiesen, die den Wettbewerb verzerren können und unter Druck seitens der Kostenträger und des Bundesrats stehen [8]. All dies könnte aus Sicht der SGAIM langfristig dazu führen, dass in der Schweiz multimorbide Patienten nicht mehr adäquat versorgt werden können.

Der Kostendeckungsgrad zeigt auf den ersten Blick paradoxerweise ein anderes Bild und kann zu Trugschlüssen bezüglich Systemgüte führen. Er scheint in der für die Systementwicklung entscheidenden Betrachtung der DRG-Sicht ausgewogen und zeigt teilweise sogar einen Trend zu höheren Werten bei DRGs mit einem hohen durchschnittlichen PCCL-Wert. Da die DRGs mit einem hohen Anteil an multimorbiden Fällen auch höhere Fallkosten aufweisen, fallen aber trotz ähnlichem Kostendeckungsgrad deutlich höhere Defizite pro Fall an.

Der Anteil von multimorbiden Fällen mit schweren und äusserst schweren Komorbiditäten und Komplikationen (PCCL 3,0 und 4,0) betrug in unserem Kollektiv knapp $20 \%$. Es handelt sich somit nicht um eine seltene Patientengruppe, und ihre ökonomische Benachteiligung ist systemrelevant.

In unseren Daten zeigt sich in den Jahren 2013 bis 2015 eine tendenzielle Verbesserung der ökonomischen Situation aller Fallkategorien mit einem deutlichen Rückschlag im Jahr 2016. Daraus kann aber nicht unbedingt ein Rückschluss auf die ökonomische Systementwicklung in der ganzen Schweiz gezogen werden, da sich das untersuchte Spitalkollektiv und damit die Datenbasis für die verschiedenen Jahre unterscheiden.

\section{Einschränkungen}

Die Lieferung der umfangreichen Daten durch die Spitäler war selbstverständlich freiwillig und wurde durch etwa die Hälfte der angefragten Häuser abgelehnt. Es könnte daher sein, dass die Daten nicht repräsentativ sind, weil eher Spitäler mit einem problematischen Geschäftsgang unter SwissDRG ihre Daten zur Verfügung gestellt haben. Die Analyse der Daten einzelner Spitäler zeigt aber, dass auch bei Häusern mit insgesamt positivem Ergebnis (Anteil je nach Datenjahr zwischen 5\% und 54\%) der reziproke Zusammenhang zwischen PCCL und Ergebnis pro Fall beobachtet wird.

Betreffend Fallsicht mag man einwenden, dass es aufgrund der systembedingten statistischen Streuung der
Fälle innerhalb der DRGs nicht immer möglich ist, die Fälle innerhalb einer DRG unabhängig vom Grad ihrer Multimorbidität zu vergüten. Wir erwarten aber, dass sich dieser Effekt durch systematische DRG-Splits vermindern lassen sollte.

\section{Ausblick}

Unsere Analysen wurden bereits Anfang 2016 mit Vertretern der SwissDRG AG diskutiert, und es wurde dem Vorstand der SGAIM zugesichert, dass die Problematik angegangen werden soll. Unserer im Rahmen des Antragsverfahrens geäusserten Bitte, unsere Resultate an den gesamten Datensätzen zu überprüfen und uns das Ergebnis mitzuteilen, wurde bisher nicht entsprochen.

Alle antragsberechtigten Partnerorganisationen können über das jährliche Antragsverfahren zur Systementwicklung beitragen, was auch die SGAIM tut. Falls sich aber die generell schlechtere finanzielle Abgeltung von komplexen Fällen bzw. DRGs mit einem hohen Anteil an multimorbiden Fällen in den Gesamtdaten der SwissDRG AG reproduzieren lässt, sollte dieses Ungleichgewicht auch von dieser Seite beseitigt werden. Über Schritte in diese Richtung, nämlich die verbesserte Abbildung aufwendiger Fälle, wurde an der Präsentation des aktuell gültigen Systems 7.0 der SwissDRG AG am 26.9.2017 mit Verweis auf die Anregungen der SGAIM berichtet.

\section{Literatur}

1 Le Reste JY, Nabbe P, Manceau B, Lygidakis C, Doerr C, Lingner H, Czachowski S, Munoz M, Argyriadou S, Claveria A, Le Floch B, Barais M, Bower P, Van Marwijk H, Van Royen P, Lietard C. The European General Practice Research Network presents a comprehensive definition of multimorbidity in family medicine and long term care, following a systematic review of relevant literature. J Am Med Dir Assoc. 2013;14(5):319-25.

2 Glynn LG, Valderas JM, Healy P, Burke E, Newell J, Gillespie P, Murphy AW. The prevalence of multimorbidity in primary care and its effect on health care utilization and cost. Fam Pract. 2011;28(5):516-23.

3 Fortin M, Bravo G, Hudon C, et al. Relationship between multimorbidity and health-related quality of life of patients in primary care. Quality of life research: an international journal of quality of life aspects of treatment, care and rehabilitation. 2006;15(1):83-91.

4 Nunes BP, Flores TR, Mielke GI, Thume E, Facchini LA. Multimorbidity and mortality in older adults: A systematic review and meta-analysis. Archives of gerontology and geriatrics. 2016;67:130-8.

5 Wolff JL, Starfield B, Anderson G. Prevalence, expenditures, and complications of multiple chronic conditions in the elderly. Arch Intern Med. 2002;162(20):2269-76.

6 Grant RW, Ashburner JM, Hong CS, Chang Y, Barry MJ, Atlas SJ. Defining patient complexity from the primary care physician's perspective: a cohort study. Ann Intern Med. 2011;155(12):797-804.

7 https://www.bfs.admin.ch/bfs/de/home/statistiken/ bevoelkerung/zukuenftige-entwicklung.assetdetail.40822.html

8 https://www.admin.ch/gov/de/start/dokumentation/ medienmitteilungen.msg-id-59773.html

\section{Danksagung}

Wir danken allen beteiligten Spitälern für das mit der Datenlieferung verbundene Vertrauen. 